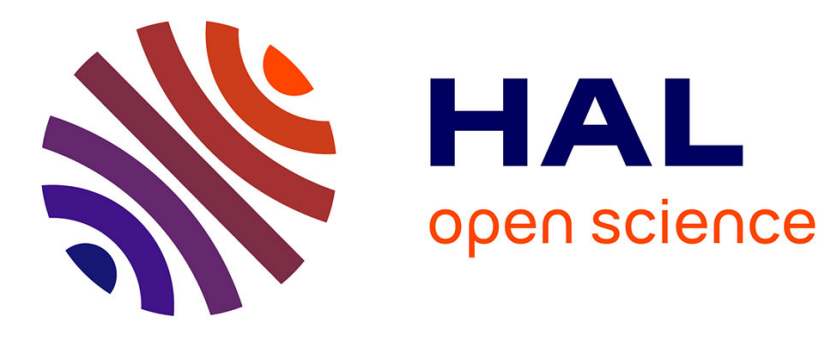

\title{
On the Use of Wireless Technologies for Shipboard Monitoring Systems
}

\author{
Hussein Kdouh, Christian Brousseau, Gheorghe Zaharia, Hanna Farhat, Guy \\ Grunfelder, Ghaïs El Zein
}

\section{- To cite this version:}

Hussein Kdouh, Christian Brousseau, Gheorghe Zaharia, Hanna Farhat, Guy Grunfelder, et al.. On the Use of Wireless Technologies for Shipboard Monitoring Systems. Wireless Personal Communications, 2013, 72 (3), pp.1755-1769. 10.1007/s11277-013-1133-9 . hal-00872320

\section{HAL Id: hal-00872320 https://hal.science/hal-00872320}

Submitted on 11 Oct 2013

HAL is a multi-disciplinary open access archive for the deposit and dissemination of scientific research documents, whether they are published or not. The documents may come from teaching and research institutions in France or abroad, or from public or private research centers.
L'archive ouverte pluridisciplinaire HAL, est destinée au dépôt et à la diffusion de documents scientifiques de niveau recherche, publiés ou non, émanant des établissements d'enseignement et de recherche français ou étrangers, des laboratoires publics ou privés. 


\title{
On the Use of Wireless Technologies for Shipboard Monitoring Systems
}

\author{
Hussein Kdouh ${ }^{1}$, Christian Brousseau ${ }^{2}$, Gheorghe Zaharia ${ }^{1}$, Hanna Farhat ${ }^{1}$, Guy Grunfelder ${ }^{1}$ \\ and Ghaïs El Zein ${ }^{1}$
}

Institut d'Electronique et de Télécommunications de Rennes, 1. INSA de Rennes, 2. Université de Rennes 1, Rennes, 35708, France

Tel: +33223238292

Fax: +33223238439

Email : 1. FirstName.LastName@insa-rennes.fr, 2. FirstName.LastName@univ-rennes1.fr This work is a part of SAPHIR project funded by "Région Bretagne". It was presented in the $14^{\text {th }}$ International Symposium on Wireless Personal Multimedia Communication (WPMC 2011) in Brest, France.

Abstract: Current shipboard monitoring systems use extensive lengths of cables to connect sensors to control units. Replacing wired connections by wireless ones may be an efficient solution to reduce the ship weight and cost. Ships are characterized by a specific metallic environment which can severely decrease the efficiency of wireless networks due to signal attenuation and multipath effects. In this paper, we present a feasibility study of a Wireless Sensor Network (WSN) using ubiquitous technologies on board vessels. A measurement campaign has been conducted on board a ferry to investigate the radio propagation challenges of wireless communications in this particular environment. Path loss models have been obtained for typical shipboard environments. Engineering rules concerning the placement and the number of communication nodes needed to cover the decks and maintain the network connectivity have been determined. Based on these results, an IEEE 802.15.4 compliant WSN has been tested on board the same ferry. Sensor nodes have been placed on the four decks of the ferry and the base station has been placed in the control room located in the bottom deck. Results show an excellent reliability with respect to transmission ratio of sensor nodes and a significant connectivity between nodes located in different compartments and decks separated by metallic watertight doors.

Keywords: Wireless sensor networks, ships, path loss models, MicaZ motes.

Abbreviations: WSN: Wireless Sensor Network; MEMS: Micro-Electro-Mechanical Systems; EM:

ElectroMagnetic; CW: Continuous Wave; ISM: Industrial Scientific and Medical; Tx: Transmitter; Rx: Receiver; dB: decibel; Minimum Mean Square Error MMSE

\section{Introduction}

Ships are an important part of modern systems widely used in armed conflict and commercial purposes such as fishing and transporting passengers and cargos. Ships manufacturers and navy companies aim to use automation on board ships as much as possible in order to improve security and to reduce the number of crew members. Modern ships are equipped with 
automatic monitoring systems which control and ensure the safety and accuracy of the whole ship operation. Current shipboard monitoring systems use extensive lengths of cables to connect several thousands of sensors to central control units. Tens of kilometers of cables may be installed on board a ferry-boat, increasing its cost, weight and architecture complexity [1]. Therefore, many research groups have started to investigate the possibility to replace the huge amount of cables by wireless connections using ubiquitous technologies. In [2], authors explore the feasibility of wireless communications for both intra- and inter-compartment shipboard communication within various naval vessels. In [3], it has been demonstrated that the radio propagation in large shipboard areas might be characterized by almost a free space law, while no path loss model has been found for corridors. In [4], authors show that rooms in below-deck spaces of a ship may be characterized as complex reverberant cavities.

Otherwise, Wireless Sensor Networks (WSN) have recently gained a worldwide attention with the great development of Micro-Electro-Mechanical Systems (MEMS) technology, which led to the development of small autonomous devices called "sensor nodes"[5]. Various WSN applications have been addressed and reported in the literature, such as industrial process monitoring [6], natural-disaster forecasting [7] and habitat monitoring [8]. Applying this technology for shipboard monitoring systems may be an efficient solution to reduce complexity and cost induced by the current wired system. However, the Electromagnetic (EM) waves propagation on board a vessel is a serious challenge. Several factors tend to decrease the performance of wireless networks in this particular environment. Metallic bulkheads, made often of steel, can severely decrease the power of received signals. Moreover, multipath effects leading to multiple delayed copies of the transmitted signal at the receiver may also decrease the radio communication data rate within ships. These characteristics make difficult the wireless communication between nodes placed in different rooms or different decks. Therefore, an EM propagation study must be performed on board a vessel before network deployment. This study is necessary in order to verify the feasibility of inter-compartments and inter-decks wireless communication and to efficiently place the nodes to have a good network connectivity. In this paper, a complete feasibility study of a WSN on board a ferry is presented. First, an EM propagation study is performed in three typical environments on board the ferry. Path loss models are determined and different communication configurations such as communication between different rooms or different decks are considered. The results of these measurements are then used to determine some engineering rules concerning the location and the number of nodes needed to ensure full radio coverage of the ship and whole network connectivity. Secondly, a WSN based on the IEEE 
802.15.4 standard is deployed on board the same ferry. Twelve sensor nodes are deployed in the four decks of the ferry and a base station constituted of a gateway node and a laptop is placed in the control room. A part of this work has been presented in [9].

The remainder of this paper is organized as follows: Section 2 describes the measurement sites. Section 3 presents the propagation study and analyses its results. Section 4 describes the WSN setup and deployment, and analyses the results of the network test. Finally, conclusions are drawn in Section 5.

\section{Measurement Sites}

The ship used for this study is a ferry boat from the 'Compagnie Océane' called 'Acadie'. The deckhouse of 'Acadie' is constituted of the following decks, arranged vertically from bottom to top: the bottom deck which houses the main engine room, the control room and the crew's cabins; the main deck which is a parking; the covered passenger deck, and the bridge deck which is constituted of the non-covered passenger deck and the wheel house. Four typical environments are considered for the propagation measurements: the engine room, the parking, the covered passenger deck and the crew's cabins. The non-covered passenger deck is similar to a classical outdoor environment. Hence, no particular measurements have been performed on this deck. However, it will be covered when deploying the WSN.

The engine room of 'Acadie' is composed of the main engine room and the control room. These two rooms are separated by a bulkhead and a watertight door, which have both a big glass window. The engine room contains engines, pumps, generators and valves. Fig. 1a shows the arrangement of the engine room of 'Acadie' where we can see the glass windows between the control room and the main engine room. The other part of the bottom deck houses the crew's cabins. This part is separated from the engine room by a thick metallic bulkhead. The cabins doors are made of wood.

The parking of 'Acadie' is constituted of a big hall with metallic walls including some glass windows and some small rooms (in the front section) with metallic watertight doors. Measurements were carried out on board the ferry when it was moored to the harbor. There were no vehicles parked within the parking. Fig. $1 \mathrm{~b}$ shows the parking where one of the metallic watertight doors used in this environment can be viewed at the right-hand side.

The covered passenger deck of 'Acadie' is a big hall with metallic walls including glass windows. It is composed of passengers' seats and tables. This environment is composite and constituted of several types of materials such as wood, glass and steel. The parking is 
connected to upper and lower decks by stairways that have a metallic watertight door on the parking side (the opened door in the right side of Fig. 1b).
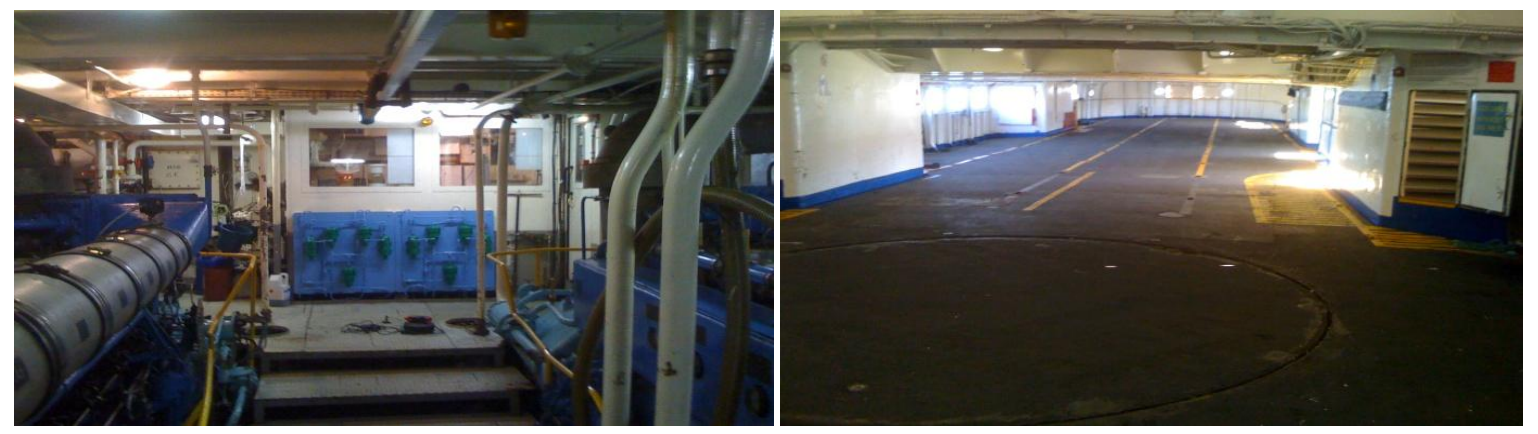

\section{Propagation Study}

This section describes the propagation measurement campaign conducted on board 'Acadie'. It includes the measurement procedure, results and analysis.

\section{Measurement Procedure}

Due to the low data rate of a shipboard WSN, Continuous Wave (CW) measurements are sufficient to characterize propagation effects related to a WSN deployment because the bandwidth of the transmitted signal is much less than the coherence bandwidth of the propagation channel. The transmission system is composed of a signal generator, an omnidirectional conical monopole antenna and some connecting cables. The signal generator delivers $0 \mathrm{dBm}$ continuous sinusoidal signal at a frequency of $2.45 \mathrm{GHz}$ (ISM radio band Industrial Scientific and Medical). The receiver is composed of a spectrum analyzer operating in a zero-span mode, a laptop to collect and save measurements data, an antenna positioner and connecting cables. We have selected the $2.4 \mathrm{GHz}$ ISM frequency band as it is used by most existing standards dedicated to WSN [5].

Each shipboard environment was measured using a standard procedure. The transmitting (Tx) antenna, which has a height of $1.80 \mathrm{~m}$, is placed at a fixed location. Path loss measurements are performed using a receiver $(\mathrm{Rx})$ with a $1.80 \mathrm{~m}$ antenna height. The receiver is placed at different locations in each shipboard environment. Tx and Rx locations are marked on a digital map to calculate the Tx-Rx separation distance. These experiments rely on the narrowband measurements of a $\mathrm{CW}$ signal at $2.45 \mathrm{GHz}$ performed to determine the path loss. The received power varies over a small area due to multipath-induced fading. However, averaging the received power values along 20 wavelength circular track using 250 power samples yields a reliable estimation of the local average power independent of signal 
bandwidth [10]. Average of the received power values in Watts is used for all path loss estimations.

\section{Measurement Results}

Fig. 2 shows the transmitter locations (Tx1 to Tx4), the receiver locations (blue squares), the layout of the ship and the measured path loss (given in $\mathrm{dB}$ in the blue squares) for each environment on board 'Acadie'.
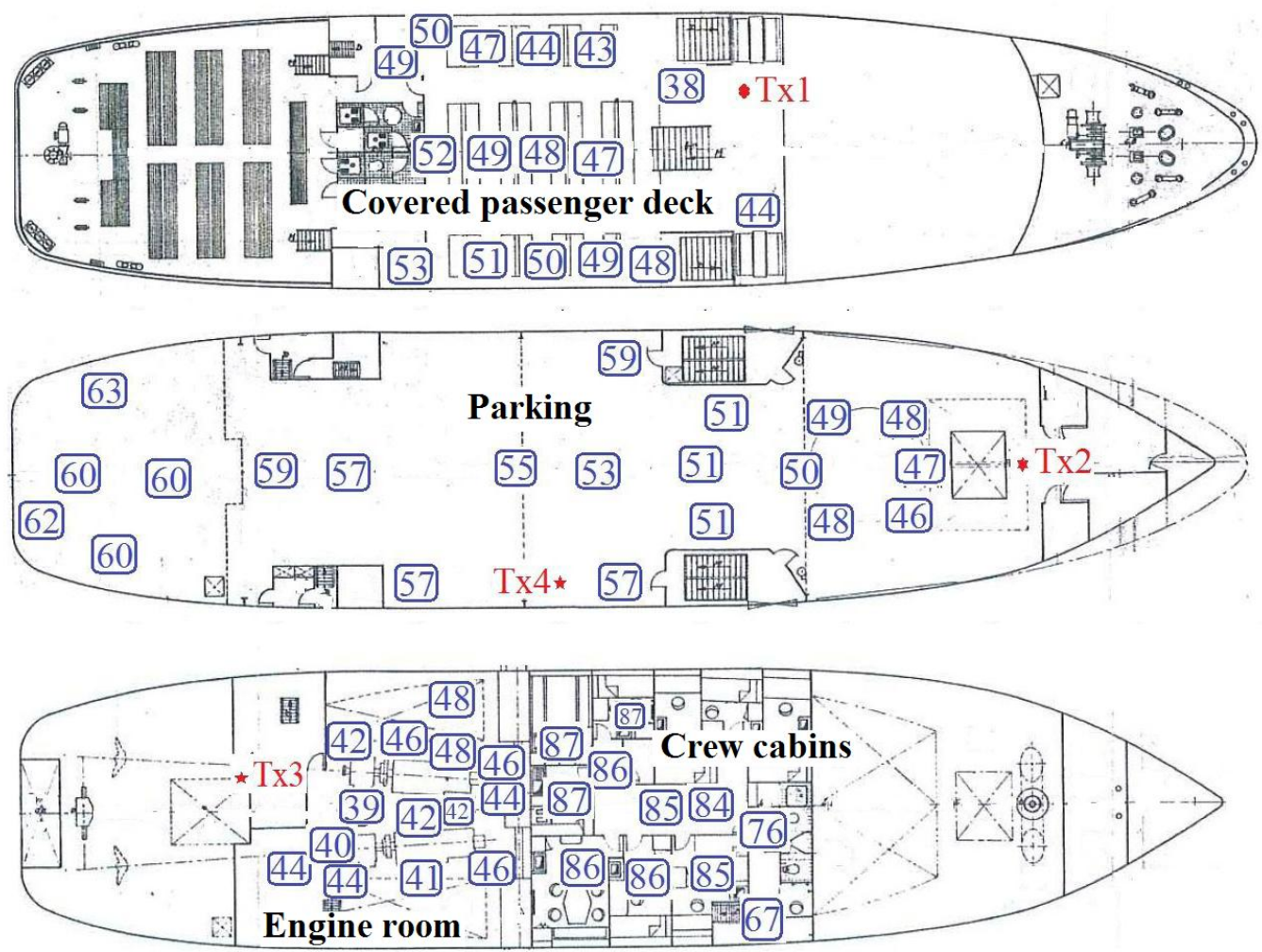

In the covered passenger deck, the transmitter was placed at the Tx1 location and the receiver was placed at 16 different locations. In the parking, the transmitter was placed at the Tx2 location and the receiver was placed at 21 different positions. In the engine room, the transmitter was placed in the control room (Tx3 location) and the receiver was placed at 14 different positions in the main engine room. To characterize the communication between decks, the transmitter was placed at the position $\mathrm{Tx} 4$ in the parking $(2 \mathrm{~m}$ in front of the watertight door) and the receiver was placed at 11 different locations in the crew cabins. These two decks are connected by metallic stairs. The entrance watertight door to the stairway in the parking was closed during these experiments. The other three stairways that connect the parking to the engine room and the passenger deck have the same architecture. The results of this experiment can be used to characterize the communication between decks on board 'Acadie'. 


\section{Results Analysis}

The three main configurations of communication between nodes are the followings:

- communication between nodes placed in the same room.

- communication between nodes placed in different rooms.

- communication between nodes placed in different decks.

A communication is considered as possible when the received power is higher than $-85 \mathrm{dBm}$. This threshold is related to the receiving sensitivity of sensor nodes that will be used later in the WSN experiment (MicaZ motes from Crossbow technology [11]).

\section{Communication between nodes within the same room}

The three considered environments in this case are: the engine room, the parking and the passenger deck. Measurement results are used to determine the relation between the path loss and the distance between nodes in each environment. Average path loss for a separation distance $d$ between the transmitter and the receiver is expressed as a function of distance by using the following expression [12]:

$$
\overline{P L(d)}=\overline{P L\left(d_{0}\right)}+10 n \log _{10}\left(\frac{d}{d_{0}}\right)
$$

where $n$ is the path loss exponent which indicates the rate at which the path loss increases with distance and $d_{0}=1 \mathrm{~m}$ is the reference distance. This model does not consider that different surrounding configurations may exist for the same Tx-Rx separation distance $d$. Measurements have shown that at any value of $d$, the path loss $P L(d)$ for a particular location is random and has a log-normal distribution around its mean distance-dependant value. Hence, path loss can be expressed as:

$$
P L(d)=\overline{P L\left(d_{0}\right)}+10 n \log _{10}\left(\frac{d}{d_{0}}\right)+X_{\sigma}
$$

where $X_{\sigma}$ is a zero-mean Gaussian distributed random variable (in dB) with standard deviation $\sigma$ (also in $\mathrm{dB}$ ). The log-normal distribution describes random effects of shadowing or multipath propagation which occur over a large number of measurement locations having the same separation distance but with different levels of clutter on the propagation paths [12]. The results of measurements performed on board the 'Acadie' vessel have shown a significant correlation with model (1). Fig. 3 shows path loss values as a function of distance for all 
environments. Shadowing effects have been taken into account by the Gaussian distributed random variable with $\sigma$ computed as the standard deviation of the error between the measurements and the model (1) results.

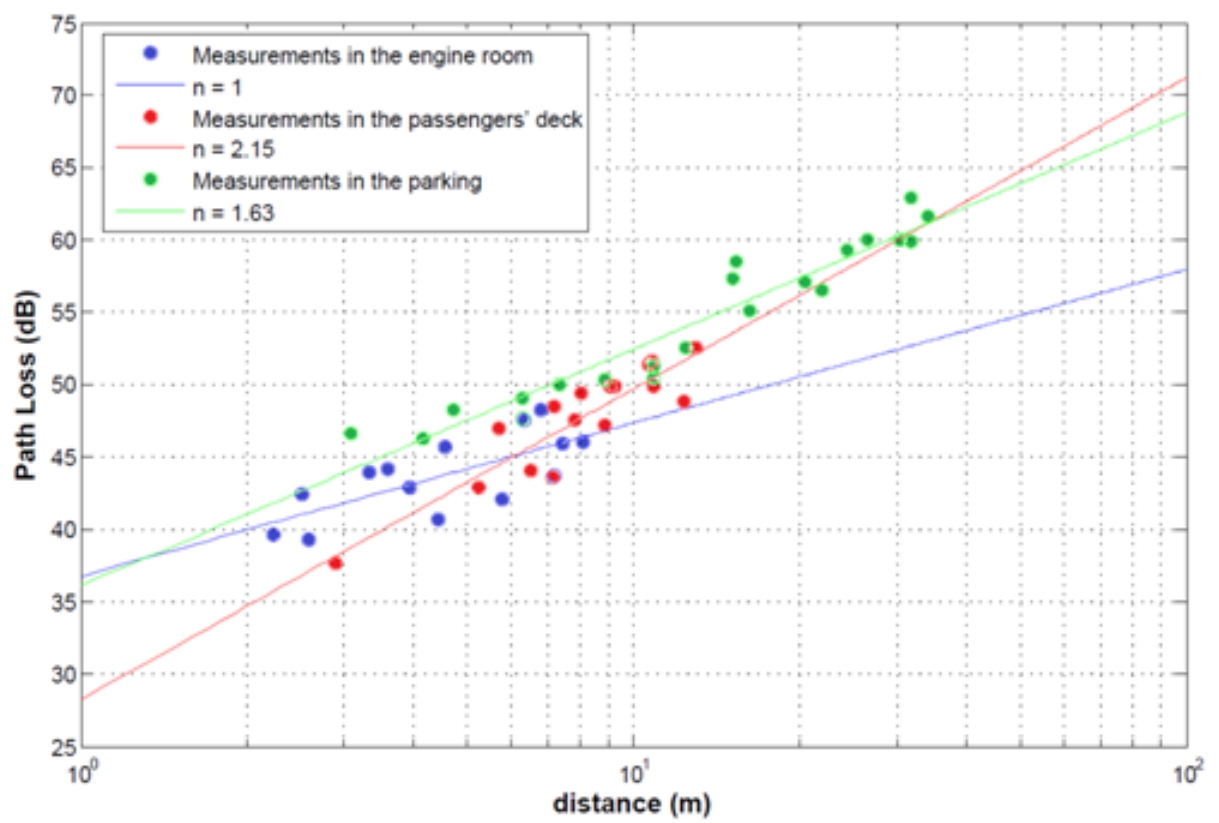

The values of $\overline{P L\left(d_{0}\right)}, n$, and $\sigma$ have been computed from measured data using linear regression (Minimum Mean Square Error MMSE estimation). Parameters of the three environments are presented in Table 1 where $r$ is the correlation coefficient between measurements and model results. The large values of $r$ show a significant correlation between measurement results and the path loss model. Nevertheless, the value of $r$ in the engine room is lower than that in other environments. This difference may be explained by the complex arrangement of metallic machines and tubes in this environment, which randomly scatters, reflects and diffracts the radio waves. The arrangement is more homogenous in the passenger deck and the parking.

\begin{tabular}{|l|l|l|l|l|}
\hline Environments & $n$ & $P L\left(d_{0}\right)$ & $\sigma$ & $r$ \\
\hline Engine room & 1 & 36.76 & 1.37 & 0.72 \\
\hline Parking & 1.63 & 36.1 & 1.21 & 0.96 \\
\hline Passenger deck & 2.15 & 28.19 & 1.25 & 0.90 \\
\hline
\end{tabular}

Some preliminary conclusions may be drawn from the values of $n$. The path loss exponent is equal to 1 in the engine room of 'Acadie'. This result can be explained by the presence of metallic walls and ceiling and the absence of significant radio leakage between the engine room and the neighborhood (the access between the engine room and the parking was closed during measurements). The transmitted energy is then kept within the engine room (waveguide behavior [13]). Moreover, the path loss exponent in the parking is equal to 1.61 
which is lower than the free space path loss exponent. This result is explained by the guiding effect of metallic walls and ceiling. However, the difference between the engine room and the parking exponents is explained by the glass windows of the parking walls which allow EM leakages for radio waves. The transmitted energy is not kept inside the parking like in the engine room where the walls are completely metallic. Furniture obstructing the visibility between Tx and Rx explain the larger value of $n$ in the covered passenger deck.

\section{Communication between nodes placed in different rooms}

The second studied configuration is the communication between nodes placed in different rooms of the same deck. EM propagation is considered in the bottom deck and the parking which contain several rooms. In this case, the propagation path between $\mathrm{Tx}$ and $\mathrm{Rx}$ is obstructed by bulkheads and doors.

The first scenario is the communication between the crew's cabins and the engine room. As stated before, these two parts are separated by a thick totally metallic bulkhead. The transmitter is located in the corridor between crew cabins and the receiver is moved in the engine room. No signal has been received, in spite of the small Tx-Rx separation distance. This is simply explained by the huge attenuation of the thick metallic bulkhead and the absence of any opening that allows EM leakage between these two adjacent areas.

The second scenario is the communication between nodes located in two adjacent rooms with a common door. Two types of doors may be considered on board 'Acadie': the metallic watertight doors that are mainly used at the entrance of stairways connecting the parking to other decks, and between small rooms located in the front section of the parking; and the wooden doors of the crew's cabins. Several experiments have been conducted to determine the excess path loss due to closing a door between two nodes, using the following experimental protocol. Tx and Rx are located in the two sides of the door and path loss is measured when the door is opened and when it is closed (with the same locations of Tx and Rx for the two cases). Excess path loss due to the door closure is determined as the difference between the two measured values. The results have shown that a metallic watertight door closure decreases the received signal by a mean value of $20 \mathrm{~dB}$ (and a standard deviation of $3 \mathrm{~dB}$ ). However, the effect of wooden doors was negligible.

\section{Communication between nodes in different decks}

Path loss levels of measurements between the parking and the passenger deck (Fig. 2) show that the transmitter located in front of the watertight door in the parking is not able to cover 
the total area of the crew's cabins deck. The maximum acceptable path loss is $85 \mathrm{~dB}$, which is less than the most of values found in this deck. Variation of path loss values in this configuration does not depend directly of the Tx-Rx separation distance. It depends on the closeness of the Rx and Tx to the stairway. This variation indicates that stairways are the main sources of EM leakage between adjacent decks. Hence, placing intermediate sensor nodes in the stairways is necessary to maintain a connectivity of shipboard WSN.

\section{Outage Probability}

Since $P L(d)$ is a random variable with a normal distribution in $\mathrm{dB}$ about the distancedependant mean so the received power at distance $d$ will be also a random variable with the same distribution. Hence, the complementary cumulative distribution function Q-function or the complementary error function $(e r f c)$ may be used to determine the probability that the received signal level will fall below a particular level. The Q-function is defined as:

$$
Q(z)=\frac{1}{\sqrt{2 \pi}} \int_{z}^{\infty} \exp \left(-\frac{x^{2}}{2}\right) d x=\frac{1}{2} \operatorname{erfc}\left(\frac{z}{\sqrt{2}}\right)
$$

The probability that the received signal level will be below the sensitivity $S$ of a sensor node can be computed as:

$$
\operatorname{Pr}\left[P_{r}(d)<S\right]=Q\left(\frac{\overline{P_{r}(d)}-S}{\sigma}\right)
$$

where $\operatorname{Pr}(d)$ is the received power at distance $d, \overline{\operatorname{Pr}(d)}$ is its mean value, and $\sigma$ is the standard deviation of $X_{\sigma} . S$ is supposed equal to $-85 \mathrm{dBm}$ for the used communication nodes [11].

For communications between two nodes located in the same room, the probability that the received power falls below $-85 \mathrm{dBm}$ is almost null for the three studied environments (engine room, parking, covered passenger deck) regardless the Tx-Rx separation distance. However, other cases may be considered in the parking depending on the number of closed watertight doors on the propagation path between Tx and Rx. Fig. 4 presents the outage probability $\operatorname{Pr}[\operatorname{Pr}(d)<S]$ as a function of the separation distance between two nodes located in the parking. For one closed watertight door between the transmitter and the receiver, the probability that the received power level is higher than $-85 \mathrm{dBm}$ exceeds $90 \%$ regardless the separation distance. However, when two watertight doors are closed between Tx and Rx, the outage probability tend to 1 when the separation distance exceeds $4 \mathrm{~m}$. Connectivity will not be possible for two nodes separated by two closed watertight door in the parking. 


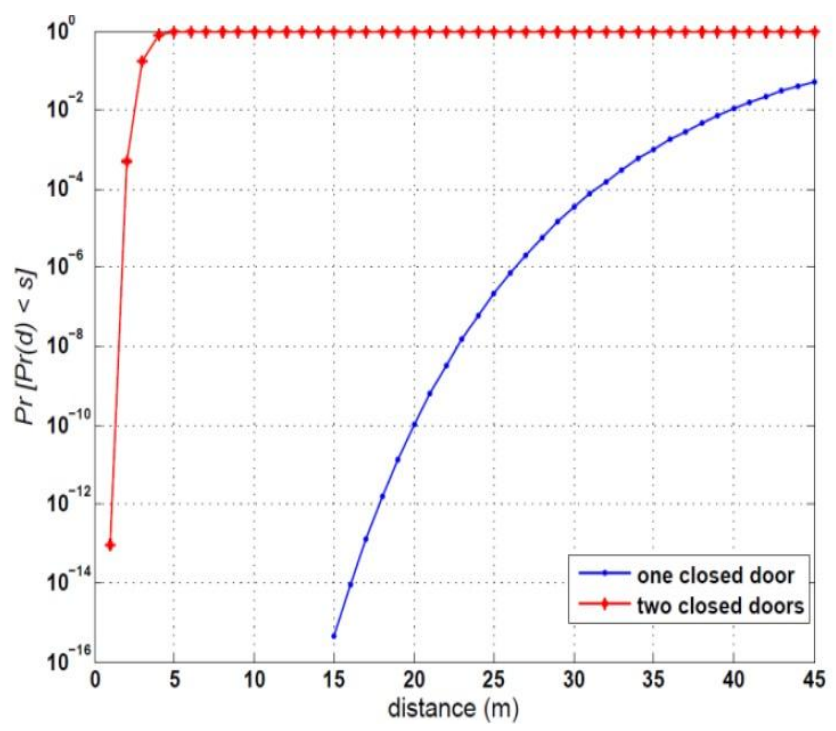

\section{Wireless Sensor Network Test}

This section describes the deployment of a WSN based on the conclusions drawn from the propagation study. In the first part of this section, the technology used in the experiment is described and then, in the second part, the deployment procedure and the obtained results are presented and discussed.

\section{Technology Used}

The shipboard WSN test was carried out using Crossbow's MICAz wireless sensor nodes (motes) [11], which are based on the 2.4 GHz IEEE 802.15.4 standard. MicaZ is a tiny wireless measurement system designed specifically for deeply embedded sensor networks. Each node is composed of a processor, an internal memory, a $2.4 \mathrm{GHz}$ radio transceiver, two $2 \mathrm{~A}$ batteries and a sensor board. It has a maximum data rate of $250 \mathrm{kbps}$. Embedded sensors can measure temperature, humidity, barometric pressure, ambient light and acceleration. Rather than developing a new routing protocol, we have decided to use the Crossbow's XMesh routing protocol which is a link-quality based dynamic routing protocol that uses periodic route update messages from each node to estimate link quality. Each node listens to the radio traffic in the neighborhood and selects the parent that would be the least costly in terms of transmissions number to reach the base station [14]. The network is composed of 12 sensor nodes and one gateway connected to a laptop via a USB cable. The laptop runs the MoteView 2.0 software which is a graphical user interface that allows visualizing the real time data sent by the WSN to the base station and the network topology evolution during the test. 


\section{Deployment Procedure}

The choice of the locations of nodes is based on the results obtained from the propagation study. Previous results have shown that EM propagation is possible between decks through stairways. To ensure the connectivity between the four decks of the shipboard WSN, relay nodes are first installed in the stairways. Hence, the deployment procedure has continued by installing (Fig. 5):

- Node 3 in the stairway between the crew's deck and the parking (the watertight door is closed)

- Node 2 in the stairway between the engine room and the parking (the watertight door is open)

- Node 7 in the stairway between the parking and the passenger deck (the watertight door is open)

- Node 11 in the stairway between the covered passenger deck and the non-covered passenger deck (this stairway has a wooden door which was closed during the test).

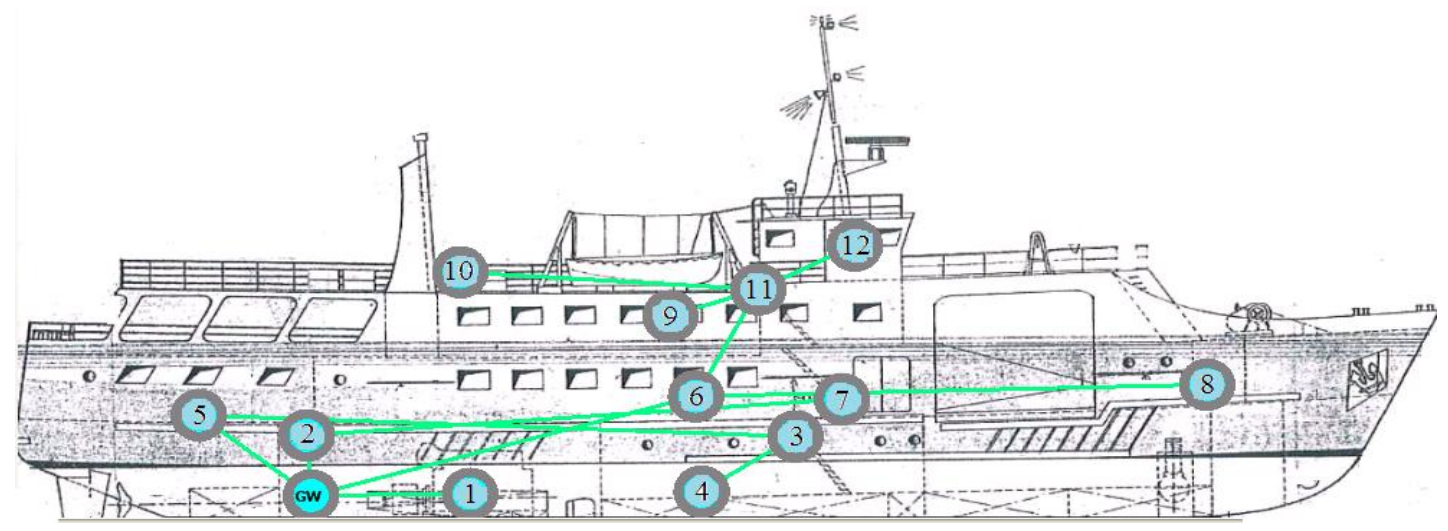

The base station is installed in the control room (same location of Tx3 in Fig. 2). As mentioned in the previous section, the outage probability is almost null in the engine room and the covered passenger deck, regardless the locations of the transmitter and the receiver. In addition to the gateway node and the relay node 7 , one node in the engine room and one node in the covered passenger deck are installed to have a good radio coverage of these rooms. Node 1 is installed on one of the two main engines in the engine room, node 4 is installed in the crew's deck and node 9, in the covered passenger deck. Node's installation becomes different in the parking where several cases have been distinguished as a function of the number of watertight doors between the transmitter and the receiver. Node 8 is installed in the small room located in the front section of the parking. The watertight door of this room is closed during the test. Node 6 is installed in the middle of the parking (below a fire sensor) and node 5 is installed in front of the second stairway located between the engine room and 
the parking. Node 12 is used to collect data from the wheel house. There are no stairs between the wheel house and the lower decks. Thus, node 10 is installed on the bridge deck as an intermediate node between the wheel house and node 11 located in the stairways between the covered and the non covered passenger deck.

\section{Network Results}

Analysis of the performance of the network has begun with the statistics of the packets sent by all the nodes during the experiment. Fig. 6 presents the percentage of originated, forwarded and dropped packets of the 12 nodes in the WSN. 'Originated' packets include all data, node health, neighbor health and route update packets originated at the node. 'Forwarded' packets are the packets that the node has forwarded from other nodes. 'Dropped' packets are the packets that the node has dropped. Packets are considered to have been dropped when 1 packet has been retransmitted 8 times without receiving the link-level acknowledgement. Obtained results (Fig. 6) show that less than $2 \%$ of packets have been dropped for most of nodes (only node 12 located in the wheel house has $7 \%$ of dropped packets due to its particular location in the wheel house separated from other ship parts). The small percentage of dropped packets reflects significant efficiency of the XMesh link-based routing protocol in such hostile environments. In Fig.6, it can be noticed that the huge amount of forwarded packets comes from the nodes 2, 3, 5, 6, 7 and 11, due to the locations of these nodes in the stairways to have the whole network connectivity. Nodes in the upper decks route their data mainly through stairway nodes, as the radio signal penetration is impossible through metallic ceiling and floors.

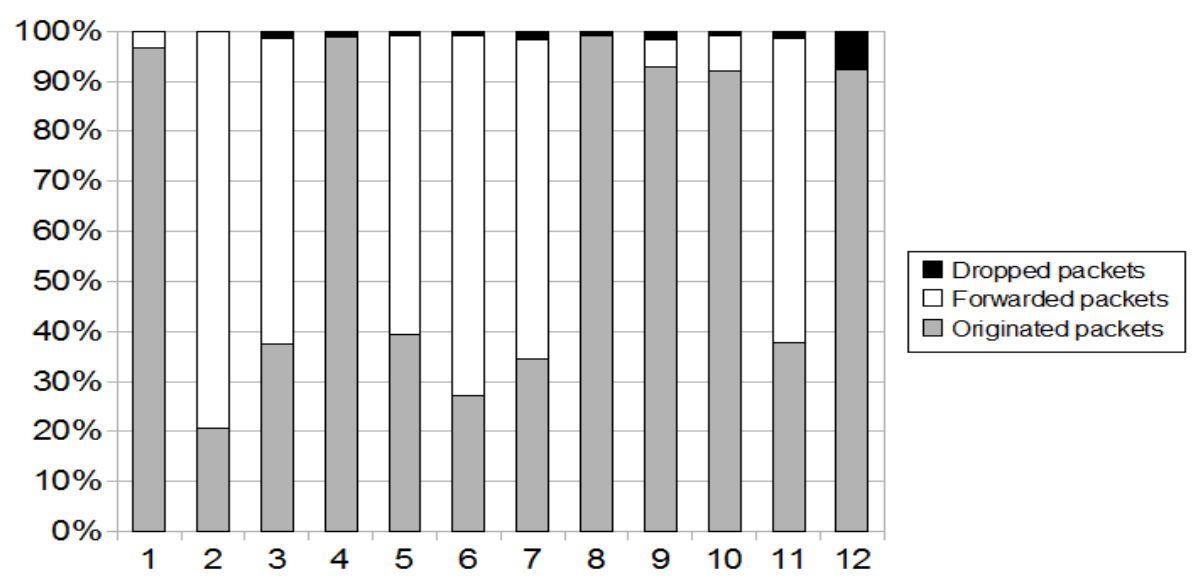

To improve knowledge about propagation inside the vessel, paths followed by packets from source nodes towards the base station have been studied. As previously stated, the sensor nodes are pre-programmed by the Crossbow XMesh routing protocol. Therefore, a sensor node selects the next hop which minimizes the number of transmissions required to send a 
packet to the base station. The selected parent node (next hop node) is then characterized by its closeness to the base station (in term of number of hops) and its good link quality with the source node. Hence, the choice of the next hop may be an indicator of the quality and stability of links between a sensor node and its one-hop neighbors. Table 2 shows the parent nodes of each mote and the percentage for each one during the test.

\begin{tabular}{|l|l|l|l|l|l|l|l|l|l|l|l|l|l|}
\hline Node & GW & 1 & 2 & 3 & 4 & 5 & 6 & 7 & 8 & 9 & 10 & 11 & 12 \\
\hline 1 & 100 & & & & & & & & & & & & \\
\hline 2 & 100 & & & & & & & & & & & & \\
\hline 3 & & & 56 & & & 34 & 9 & 1 & & & & & \\
\hline 4 & & & & 100 & & & & & & & & & \\
\hline 5 & 65 & & 35 & & & & & & & & & & \\
\hline 6 & 65 & & 35 & & & & & & & & & & \\
\hline 7 & & & 33 & 10 & & 42 & 15 & & & & & & \\
\hline 8 & & & & & & 1 & 99 & & & & & & \\
\hline 9 & & & & & & & 45 & 43 & & & & 12 & \\
\hline 10 & & & & & & & & 49 & & & & 51 & \\
\hline 11 & & & & & & & 70 & 27 & & 3 & & & \\
\hline 12 & & & & & & & & & & & 18 & 82 & \\
\hline
\end{tabular}

Several remarks may be done from this table. It can be noticed that node 8 has never selected node 3 (or node 7) as parent node despite the small distance between them. This behavior is in agreement with the statement that two nodes separated by two closed watertight doors cannot be connected in the parking (nodes 3 and 7 are located in two stairways with closed doors). However, this connection remains possible when only one closed watertight door separates the two nodes (which is the case of nodes 6 and 8). Furthermore, it can be noticed that nodes 1 and 2 were directly connected to the base station during the test. This was expected, as these three nodes are located in the engine room where the probability of outage between two nodes is almost null. Node 4 has always node 3 as parent node. Connection between the crew's deck and the parking is not possible because the watertight door in the entrance of the parking is closed. In spite of the small distance between nodes 4 and 1, the connection between them is impossible since the engine room and the crew's deck are separated by a thick metallic bulkhead. However, it can be noticed that node 6 located in the middle of the parking is directly connected to the base station for $65 \%$ of the time (as well as node 5) and node 9 is connected to node 6 for $45 \%$ of the time. This can be explained by the fact that the two watertight doors of the first stairway between the engine room and the parking and the first 
stairway between the parking and the passenger deck are opened. These two nodes used the intermediate nodes located in the stairways (nodes 7 and 2) for the remaining time when the direct connection becomes impossible. Finally, node 12 located in the wheel house has node $11(82 \%)$ and node $10(18 \%)$ as parent nodes. The direct connection between 12 and 11 is probably provided by the signal reflection on the metallic tour upside the non-covered passenger deck (near to node 10 in Fig. 5).

\section{Conclusions}

In this paper, results of measurement campaigns conducted on board a ferry boat are presented. The EM propagation is characterized within the $2.4 \mathrm{GHz}$ ISM frequency band. Several environments on board the ferry are considered and the effect of closing hatches on the link quality is determined. Additional measurements are carried out to characterize the wireless communication between decks. Based on these measurements, a path loss model is proposed and used to estimate the number of nodes needed on each deck. A WSN using the IEEE 802.15.4 standard and the Crossbow XMesh protocol was installed on board the ferry. This network showed a very good connectivity during the test. Thanks to this study, the feasibility of ubiquitous wireless technologies in shipboard monitoring systems has been verified.

\section{Acknowledgments}

The authors thank Marinelec Technologies and Compagnie Océane for the opportunity to conduct these measurements campaigns on board the 'Acadie' ferry.

\section{References}

[1] J. P. Lynch, and K. J. Loh. A summary review of wireless sensors and sensor networks for structural health monitoring. The Shock and Vibration Digest, 2006, Vol. 38, No. 2, pp.91-128.

[2] D. Estes, T. B. Welch, A. A. Sarkady, and H. Whitesel. Shipboard radio frequency propagation measurements for wireless networks. MILCOM2001 - Military Communications Conference, McLean, VA, USA, Oct. 2001.

[3] A. Mariscotti, M. Sassi, A. Qualizza, and M. Lenardon. On the propagation of wireless signals on board ships. I2MTC - IEEE Instrumentation and Measurement Technology Conference, Austin, TX, USA, May 2010. [4] G. B. Tait, and M. B. Slocum. Electromagnetic environment characterization of below-deck spaces in ships. IEEE International Symposium in Electromagnetic Compatibility, Detroit, MI, USA, August 2008.

[5] J. Yick, B. Mukkerjee, and D. Ghosal. Wireless sensor network survey. Computer Networks, 2008 , Vol. 52, No.12, pp. 2292-2330. 
Wireless sensor network for substation monitoring: Design and deployment. SenSys'08 - 6th Intl. Conf. on Embedded Networked Sensor Systems, Raleigh, NC, USA, Nov. 2008, pp. 365-366.

[7] G. Werner-Allen, K. Lorincz, J. Johnson, J. Lees, and M. Welsh. fidelity and yield in a volcano monitoring sensor network. OSDI '06 - 7th USENIX Symposium on Operating systems design and implementation, Seattle, WA, USA, Nov. 2006, pp. 381-396.

[8] R. Szewczyk, A. Mainwaring, J. Polastre, J. Anderson, and D. Culler. An analysis of a large scale habitat monitoring application. SenSys'04 - 2nd Intl. Conf. on Embedded Networked Sensor Systems, New York, NI, USA, Dec. 2004, pp. 214-226.

[9] H. Kdouh, C. Brousseau, G. Zaharia, G. Grunfelder, and G. El Zein. Applying ubiquitous wireless technologies for shipboard monitoring systems. WPMC11 - IEEE International symposium on wireless personal multimedia communications, Brest, France, 2011.

[10] G. Durgin, T. S. Rappaport, and X. Xu. Measurements and models for radio path loss and penetration loss in and around homes and trees at 5.85 GHz. IEEE Transactions on Communications, Nov. 1998, Vol. 46, No. 11, pp. 1484 - 1496.

[11] Memsic Technology, "MicaZ motes datasheet". http://www.memsic.com/support/documentation/wireless-sensor-networks/category/7-datasheets.html

[12] T. S. Rappaport. Wireless Communications: Principles and Practice. Prentice Hall, 2002.

[13] B. Sklar. Rayleigh fading channels in mobile digital communications systems - Part 1 : Characterization. IEEE Communications Magazine, July 1997.

[14] Memsic Technology, "Xmesh user's manual". http://www.memsic.com/support/documentation/wireless-sensor-networks/category/6-user-manuals.html

\section{Figure Legends}

Fig. 1 View of the rooms of 'Acadie' (a. View of the engine room, b. View of the parking )

Fig. 2 Layout of different parts of the 'Acadie' vessel, and locations of the transmitter Tx1, Tx2, Tx3 and Tx4 (in red), and the receivers (blue squares). Values in the blue squares are the path loss in $\mathrm{dB}$

Fig. 3 Scatter plot of path loss versus Tx-Rx distance within the same room

Fig. 4 Outage probability in the parking as a function of the separation distance and the number of closed watertight doors between the transmitter and the receiver

Fig. 5 Topology of the wireless sensor network deployed on board 'Acadie' vessel

Fig. 6 Packets statistics of the WSN nodes deployed on board 'Acadie' vessel

\section{Table Titles}

Table 1 Path loss parameters

Table 2 Parent time rate (in \%) for all sensor nodes in the network 